\title{
A Review of Current Tools Used for Evaluating the Severity of Obstructive Sleep Apnea
}

This article was published in the following Dove Press journal:

Nature and Science of Sleep

\author{
Wenhao Cao (ID) \\ Jinmei Luo \\ Yi Xiao
}

Department of Respiratory Medicine, Peking Union Medical College Hospital, Chinese Academy of Medical Sciences \& Peking Union Medical College, Beijing, People's Republic of China
Correspondence: Yi Xiao

Department of Respiratory Medicine,

Peking Union Medical College Hospital,

Chinese Academy of Medical Sciences \&

Peking Union Medical College, Beijing,

People's Republic of China

Email xiaoyipumch@sina.com

\begin{abstract}
Obstructive sleep apnea (OSA) is a common and heterogeneous disease characterized by episodic collapse within the upper airways, which leads to reduced ventilation and adverse consequences, including hypoxia, hypercapnia, sleep fragmentation, and long-term effects such as cardiovascular comorbidities. The clinical diagnosis of OSA and its severity classification are often determined based on the apnea-hypopnea index (AHI), defining the number of apneic and hypopnea events per hour of sleep. However, the limitations of the AHI to assess disease severity have necessitated the exploration of other metrics for additional information to reflect the complexity of OSA. Novel parameters such as the hypoxic burden have the potential to better capture the main features of OSA by maximizing the information available from the polysomnogram. These emerging measures have described multidimensional qualities of sleep-disordered breathing events and breathing irregularity and will ultimately result in better management of OSA.
\end{abstract}

Keywords: obstructive sleep apnea, apnea-hypopnea index, disease severity, hypoxic burden, polysomnogram

\section{Introduction}

Obstructive sleep apnea (OSA) is a highly prevalent and heterogeneous disorder characterized by repeated pharyngeal collapse, resulting in episodic reduced ventilation that causes blood gas exchanges to be disrupted and subsequent hypoxia, hypercapnia, and sleep fragmentation. ${ }^{1-3}$ Global prevalence data show almost 1 billion patients with OSA, ${ }^{4}$ predominantly in China, followed by North and South American countries, such as the United States and Brazil. The total cost of OSA in the United States in 2015 was 12.4 billion dollars, whereas data from resource-poor countries have not yet been collated. Among the many health concerns inherent to OSA, comorbidities such as cardiometabolic disorders and neurocognitive complications are the long-term consequences of the lack of precise management strategies for OSA. ${ }^{5}$ The high prevalence and socioeconomic impact of this disorder necessitate that all nations - both resource-rich and developing countries - make substantial efforts to address this issue. ${ }^{6,7}$ The current goal for healthcare systems internationally is to concentrate on providing universal diagnostic tools and effective treatment for OSA to achieve a positive impact on global health. ${ }^{8}$

As the understanding of physiological characteristics of sleep disturbance has advanced, the apnea-hypopnea index (AHI) is the tool used most commonly to diagnose and categorize the disease severity of OSA, and use of the AHI is a common practice in most studies. ${ }^{9-12}$ However, the AHI has several important limitations: poor correlation of the AHI with clinical manifestations of OSA such as excessive daytime sleepiness, which may be measured both subjectively and objectively; ${ }^{13,14}$ consequences resulting from the 
cardiovascular disease (CVD) risks that cannot be predicted precisely by the $\mathrm{AHI} ;{ }^{15}$ and the increased risk of hypertension in patients with OSA with a higher AHI score, but no further positive relationship for an AHI score $>15 .{ }^{16}$ All of these weaknesses lead to questioning the extensive use of this metric as a major strategy to evaluate OSA. ${ }^{17,18}$

The poor performance of the AHI may result from it lacking the capacity to reflect the respiratory event duration and extent, the arousal threshold, sleep fragmentation, and other pathophysiological elements. For example, a respiratory event with a 2-minute duration has a totally different physiological impact on patients with OSA than events lasting only 20 seconds. Thus, limited information provided by the AHI may be the reason that a large clinical trial (the Sleep Apnea cardiovascular Endpoints trial) showed no CVD benefit from treatment with continuous positive airway pressure (CPAP) among individuals selected based on the AHI score, ${ }^{19,20}$ although the ASAP-HF Pilot Trial compared standard of care therapy for acute decompensated heart failure versus addition of PAP therapy in patients with concomitant OSA, and found pulmonary hypertension was reduced with addition of PAP therapy. ${ }^{21}$

Undoubtedly, OSA has a strong relationship with diverse poor health outcomes, including cardiometabolic events, systemic hypertension, neurocognitive impairment, and all-cause mortality. $^{22-24}$ Intense disagreements have arisen within the science community on whether the diagnosis and treatment of this complex disease should be based solely on one parameter that displays only apnea and hypopnea polysomnogram (PSG) frequencies to the exclusion of more revealing data, such as the duration, magnitude, and distributions of oxygen desaturation in different sleep stages. Nevertheless, these other sleep traits have not been fully used in various versions of clinical practice guidelines or for expert consensus. ${ }^{25}$

This review aims to summarize the existing and emerging methods that quantify the severity of OSA beyond the data provided by the AHI score, based on a comprehensive exploration of the literature (Table 1), and to establish a broader vision to understand the pathophysiological diversity of OSA and its various susceptibilities.

\section{Conventional Methods for PSG Data Analysis Lowest Oxygen Saturation, Time Spent with Oxygen Saturation $<90 \%$, and Oxygen Desaturation Index}

Obtaining just AHI from a PSG containing rich physiological data is as unacceptable as getting just the details of forced expiratory volume in one second (FEV1) from a complicated lung function test report, thus concern is growing regarding the loss of capturing other data. This

Table I Polysomnographic Metrics of Measuring Severity of OSA

\begin{tabular}{|c|c|c|c|}
\hline Classification & Metrics & Pathophysiological Backgrounds & Complications Associated with OSA \\
\hline \multirow{3}{*}{$\begin{array}{l}\text { Conventional and } \\
\text { widely used }\end{array}$} & $\mathrm{AHI}$ & \multirow[t]{3}{*}{ intermittent hypoxia } & EDS, ${ }^{13,14}$ CVD and all-cause mortality, ${ }^{15}$ hypertension ${ }^{16}$ \\
\hline & ODI, T90, LSpO2 & & $\begin{array}{l}\text { subclinical atherosclerosis, }{ }^{25} \text { all-cause mortality in } \mathrm{HF}^{26} \\
\text { postoperative complications }\end{array}$ \\
\hline & SIT & & hypoxemia in sleep disorders ${ }^{34}$ \\
\hline \multirow{3}{*}{$\begin{array}{l}\text { Novel and } \\
\text { promising }\end{array}$} & Hypoxic burden & \multirow[t]{3}{*}{ intermittent hypoxia } & CVD mortality, ${ }^{15} \mathrm{BP}^{35}$ and risk of incident $\mathrm{HF}^{36}$ \\
\hline & Obstruction severity & & CVD and all-cause mortality ${ }^{42}$ \\
\hline & hypoxia load & & CVD risk, ${ }^{44} \mathrm{BP}^{45}$ \\
\hline \multirow[t]{4}{*}{$\begin{array}{l}\text { Emerging and } \\
\text { potential }\end{array}$} & $\begin{array}{l}\text { ApEn of oxygen } \\
\text { saturation }\end{array}$ & $\begin{array}{l}\text { indirect metric, mainly quantification } \\
\text { of data regularity }\end{array}$ & \multirow[t]{2}{*}{ Hypoxemia, ${ }^{48,49}$ Associated with $\mathrm{AHI}{ }^{60}$} \\
\hline & flow:drive ratio & pharyngeal obstruction & \\
\hline & CPC, HRV, ORP & sympathetic activation, arousability & effect of CPAP titration, ${ }^{54,58}$ CVD risk ${ }^{52,57}$ \\
\hline & $\begin{array}{l}\text { Expiratory time } \\
\text { constant }\end{array}$ & $\begin{array}{l}\text { product of airway resistance and } \\
\text { lung compliance }\end{array}$ & severe sleep apnea ${ }^{61}$ \\
\hline
\end{tabular}

Abbreviations: OSA, obstructive sleep apnea; AHI, apnea-hypopnea index; ODI, oxygen desaturation index; T90, time spent with oxygen saturation <90\%; LSpO2, lowest oxygen saturation; SIT, saturation impairment time; ApEn, approximate entropy; CPC, Cardiopulmonary coupling; HRV, heart rate variability; ORP, odds ratio product; EDS, excessive daytime sleepiness; CVD, cardiovascular disease; HF, heart failure; BP, blood pressure; CPAP, continuous positive airway pressure. 
information includes an evaluation of nocturnal hypoxemia related to apnea and hypopnea, such as the time spent with oxygen saturation $<90 \%$ (T90) or $80 \%$ (T80), the lowest oxygen saturation $\left(\mathrm{LSpO}_{2}\right)$, and the oxygen desaturation index (ODI), which is calculated as the frequency of desaturation events that is determined by $a \geq 3 \%$ or $4 \%$ decrease in peripheral oxygen saturation $\left(\mathrm{SpO}_{2}\right)$ from the baseline.

Several observational studies ${ }^{26-33}$ have demonstrated these oxygen measures to be effective or even superior to the AHI in predicting adverse CVD outcomes and all-cause mortality. For example, more serious hypoxemia (defined as T90 $\geq 0.64 \%$ ) was associated with subclinical atherosclerosis when crudely regarding T90 as a dichotomized variable. ${ }^{26}$ Another study indicated that T90 was independently associated with increased all-cause mortality in patients with heart failure as a chronic stable condition. ${ }^{27}$ A study claimed that hypoxemic burden measured by T90 was demonstrated to be more predictive for mortality than AHI and should be considered a key metric for therapies used to treat central sleep apnea. ${ }^{28}$ Previous studies revealed that oximetry parameters, including T90, ODI, and $\mathrm{LSpO}_{2}$, may play a role in predicting the postoperative complications after upper airway operations, bariatric surgery, and cardiac surgery in patients with $\mathrm{OSA}^{29-31}$ and may provide information for risk stratification. A study exploring the relationship between OSA and diabetes-related complications showed that ODI was associated significantly with a decline in estimated glomerular filtration, ${ }^{32}$ whereas another study of patients with OSA and diabetes mellitus found a relationship between $\mathrm{LSpO}_{2}$ and hyperglycemia. ${ }^{33}$

An important caution is that these metrics derived from desaturation signals related to corresponding respiratory events are not specific to OSA causing intermittent hypoxemia-they also characterize chronic airway diseases, such as chronic obstructive pulmonary disease, leading to persistent hypoxemia. In addition, whether the specific cut-off point of oxygen desaturation should be $3 \%$ or $4 \%$ remains controversial to some extent, because a different definition results in a significant variation in findings. ${ }^{34}$

Another weakness of these metrics in terms of application is that the diagnostic and classification thresholds seem arbitrary, such as ODI3\% and ODI4\%, T90 $\geq 0.64 \%$, and T90 $\geq 10 \% .^{20,26,34}$ In addition, an inherent limitation of these derived variables is that they focus only on some of the major pathophysiological traits of OSA - oxygen duration, extent, and frequency - and inevitably ignore other elements, thus falling short of capturing the overall features of OSA and eventually failing to serve as a perfect marker in clinical practice.

\section{Saturation Impairment Time}

An early established parameter, the saturation impairment time (SIT) index, was calculated as an area under the desaturation curve by integrating the time and degree of desaturation below certain levels. ${ }^{35}$ A study comparing the validity of the SIT index and time spent with oxygen saturation lower than various levels such as $90 \%$ (T90) or $80 \%$ (T80) for the quantitative evaluation of hypoxemia in sleep disorders, showed that the SIT score correlated well with T90 and T80. These results suggest that the SIT index may provide additional quantitative information to determine the severity of hypoxemia beyond the AHI. ${ }^{36}$ Nevertheless, the use of the SIT index was assumed to have approximately the same inherent defects as T90 or ODI, and thus, to date, only limited literature is available for the SIT index, resulting in insufficient advances in this field.

\section{Novel and Promising Parameters in Reinventing the Use of PSG Hypoxic Burden}

A recent study exhibited a novel parameter termed the hypoxic burden, which is determined as the average area under the desaturation curve associated with each respiratory event. ${ }^{15}$ The area under the pre-event baseline was calculated within a specific search window obtained from overlying oximetry signals with respect to the end of each oxygen desaturation event. These data can be visualized as a triangle, with the desaturation duration and depth assumed to be the base and height, respectively. The hypoxic burden can then be approximately equal to the multiplication of the AHI score and the area above the desaturation curve, thus achieving an integration of the duration, depth, and frequency of respiratory-related events. The hypoxic burden was demonstrated to be associated with an increased CVD mortality among adults aged $>40$ years in two cohort studies (the Outcomes of Sleep Disorders in Older Men [MrOS] and the Sleep Heart Health Study [SHHS]) adjusted for multiple covariates, including the AHI. ${ }^{15}$ In addition, a higher blood pressure $^{37}$ and the risk of incident heart failure in men ${ }^{38}$ were demonstrated to be associated with this hypoxic burden after adjusting for confounding factors such as comorbidities.

Compared with a single-trait measure such as the AHI score, this emerging algorithm to quantify the hypoxemia 
burden has provided a novel solution to measure the ventilatory disturbance and has made great progress in solving the mystery of the pathophysiology and multisystem outcomes of sleep-disordered breathing. ${ }^{39}$ In addition, the hypoxic burden may serve to identify patients who may benefit from CPAP and bariatric surgery. ${ }^{15} \mathrm{~A}$ limitation of the hypoxic burden identified was that while apnea and hypopnea events have been identified on sleep measures, no distinction was ever made between the obstructive and central sleep disturbances regardless of associated oxygen desaturation, and obstructive apnea and hypopnea were incorporated with equal weights. In addition, the algorithm of the hypoxic burden does not reflect the duration of respiratory events, which is a weakness of this sleep measure because it is unclear whether these impressive findings would remain significant after considering the event duration. This point is an important consideration because a study has already indicated that respiratory events of short duration may be more effective in predicting allcause mortality than longer-duration events. ${ }^{40}$

\section{Obstruction Severity}

Similar to the hypoxic burden, a novel parameter termed obstruction severity is determined as a sum of the product of each respiratory event-associated area above the desaturation curve and the duration of the corresponding apneic or hypopnea event, then normalized with the total sleep time. ${ }^{41}$ Where this metric differs from the hypoxic burden is that it considers the obstruction duration; thus, it captures a wider range of pathophysiological traits, including both the duration and the frequency of obstruction and desaturation events, plus the depth of desaturation, making it a virtually better measure with preferable prospects for application. ${ }^{42}$ Based on the results of assessment for obstruction severity, Muraja-Murro et al found that the values for this derived variable were higher in the deceased and in patients with severe OSA with an AHI score $\geq 30$ versus the control group of alive patients with matched AHI scores. Using multiple logistic regression analysis, these findings demonstrated that obstruction severity — not the AHI — was the only index related to allcause mortality in patients with severe OSA. ${ }^{43}$ In addition, the decrease in obstruction severity was as not remarkable as the decrease in the AHI score when treating patients with OSA with weight loss, which suggests that weight loss may not be an intervention that is as significant as the AHI indicates to reduce the severity of OSA. ${ }^{44}$ Thus, the dependence on a single sleep metric such as the AHI to judge the efficacy of weight loss to manage OSA may present the risk of overestimation. For better clinical practice, the obstruction severity was further converted to an adjusted AHI score to advance its use for diagnosis and as a severity measure of OSA with the same classification threshold as the conventional AHI. ${ }^{45}$ With this approach, the adjusted AHI led to a significant redistribution of OSA severity, causing a higher risk of CVD and all-cause mortality in patients with OSA with moderate and severe disease versus patients with the same severity categories based on the conventional AHI. Therefore, the methodadjusted AHI serves as a better metric to provide additional and more accurate information than the conventional AHI score alone in recognizing the risk of OSArelated complications such as CVD and all-cause mortality. ${ }^{45}$

To further clarify the definition of obstruction severity, the end of respiratory event-associated desaturation was determined as the area up to the last moment before the recovery of oxygen saturation. The ventilatory disturbance is better characterized if both the desaturation and recovery periods were incorporated in the obstruction severity calculation. In addition, the hypopnea events calculated in obstruction severity were scored following the rule that only $\geq 4 \%$ desaturation from the baseline met the criteria, leaving a smaller degree of desaturation excluded. ${ }^{43,46}$ However, the risk of self-reported CVD has been suggested to be related more to hypopnea with $\geq 4 \%$ desaturation than $<4 \%$ desaturation. ${ }^{5}$ Thus, the obstruction severity may result in a modest underestimation of disease severity in OSA.

\section{Hypoxia Load}

A third novel parameter termed hypoxia load was designed to measure the nocturnal hypoxemic burden to better classify the severity of OSA. This sleep measure was used in a prospective observational study ${ }^{47}$ showing a crude correlation between the hypoxia load-but not the AHI as an event-based metric - and the epicardial fat volume determined as a marker of CVD risk, although a causal effect has not yet been established. The hypoxia load was defined as a sum of the integrated area of all desaturation events using a trapezoidal rule, which differs slightly from the algorithm for the hypoxic burden as described previously. The hypoxia load was also demonstrated to be a promising marker of and a potential treatment target for blood pressure, ${ }^{48}$ which suggests that further studies are 
expected to illustrate the effect of the hypoxia load as a severity measure in predicting OSA outcomes.

In general, these new parameters, with a different perspective on PSG data, may provide more comprehensive information related to sleep-disordered breathing so that the intrinsic property of the physiological stress in OSA may be better assessed. However, their current use is limited by some details awaiting more evidence before reaching a consensus.

\section{Emerging Potential Markers without Specific Quantification Approximate Entropy}

A new method called approximate entropy (ApEn), which is based on chaos theory, was evaluated in several clinical studies on CVD complications. The ApEn approach represents a quantification of biological regularity in time series data. ${ }^{49}$ A smaller ApEn value corresponds to regularity and predictability, whereas a larger value indicates complexity and variability. In a recent study, ${ }^{50}$ Nakayama et al suggested that an ApEn value of chest respiratory movements was associated with a supine AHI score in patients with OSA with multiple system atrophy. In another study, the ApEn of oxygen saturation was demonstrated to be related to the AHI, ODI, and T90. ${ }^{51}$ In addition, a study on the correlation between the ApEn of oxygen saturation and the AHI based on analysis of oximetric data concluded that ApEn may be a useful approach in diagnosing OSA. ${ }^{52}$ Moreover, studies on ventilatory instability in $\mathrm{OSA}^{53,54}$ revealed that breathing irregularity may be an outcome of sleep-disordered breathing, because breathing instability improved after treating patients with OSA with CPAP. These findings suggest that ApEn may be a promising method for clinical assessment and as a target for management, especially with CPAP. Despite the substantial strength of this method, ApEn has the limitation that none of the physiological features of OSA, such as duration, frequency, and extent of desaturation, were considered when this new index was calculated. Before use in clinical practice, issues regarding the accuracy, practicality, and other aspects of ApEn remain to be resolved.

\section{Cardiopulmonary Coupling, Heart Rate Variability, and Odds Ratio Product}

An electrocardiogram (ECG)-based method has raised extensive attention after use in many studies to characterize the coupling of heart rate variability (HRV) and ECG- derived respiratory fluctuations and quantify the relative sleep quality as stable (high-frequency coupling, HFC) or unstable (low-frequency coupling, LFC), thus termed cardiopulmonary coupling (CPC). ${ }^{55}$ This measure was proven to be correlated with objective sleep quality. ${ }^{56}$ In addition, in one study, an increased HFC and decreased LFC were seen in a CPAP group, but not in the matched control group, ${ }^{57}$ suggesting that $\mathrm{CPC}$ plays a potential role in reflecting and monitoring therapeutic effects for patients with OSA. Also, CPC may be a promising marker of measuring severity and a target for treatment response.

The HRV, defined as the fluctuation of RR intervals on an ECG, reflects the autonomic variation in the nervous system. ${ }^{58}$ The time domain and frequency domain of HRV can be used to analyze autonomic nervous system activity. Abnormal HRV occurs when the autonomic nervous system function is impaired during OSA. ${ }^{59}$ Analysis of HRV showed that abnormal HRV predisposes the patient to CVDs, ${ }^{60}$ such as acute myocardial infarction. A recent study found that, compared with patients without OSA, the time domain of HRV decreases and the frequency domain increases in patients with OSA, and CPAP treatment reversed these changes in HRV. Therefore, the study concluded that HRV may help diagnose OSA and evaluate the effectiveness of CPAP treatment. ${ }^{61}$

Finally, the odds ratio product (ORP), determined as a continuous index of measuring the sleep depth extrapolated from the spectrum of an electroencephalogram (EEG), has correlations with arousability. ${ }^{62}$ In another study, compared with participants with moderate-tosevere or no OSA, higher rapid eye movement (REM) ORP was more strongly (and negatively) associated with systolic blood pressure. ${ }^{37}$ Thus, ORP may serve as a potential marker for CVD risk in patients with OSA. ${ }^{55}$

However, the CPC, HRV, and ORP, as well as some other newly developing techniques, still need to be strengthened because of their lack of specific criteria for diagnosis, severity evaluation, and indications for managing OSA.

\section{Flow:Drive Ratio}

One study quantified the severity of pharyngeal obstruction by using a continuous variable termed the flow:drive ratio, based on the features of flow shape in an individual breath during PSG. ${ }^{63}$ This feature varied widely among patients with OSA independent of the AHI score, although an association was observed between flow:drive and the AHI. Considering that this emerging metric concentrates 
mostly on the morphology of OSA without considering its major pathophysiological mechanisms, the role of the flow:drive ratio in the sequelae of OSA remains to be explored.

\section{Expiratory Time Constant}

The expiratory time constant (ETC), which reflects the tempo of pulmonary emptying in the lung mechanics, was captured based on the nasal pressure signal in PSG for patients with OSA with the overlap syndrome. ${ }^{64}$ The AHI score was greater for patients with an ETC $>0.5$ seconds than patients who had an ETC $\leq 0.5$ seconds. A larger ETC value was associated with an approximately 11 -fold increase in the risk of severe sleep apnea (odds ratio 10.6, 95\% confidence interval 3.9-51.1, $p=0.005$ ), suggesting a role for this metric to be used in the classification and management of OSA. Further research is warranted to explore more specific applications in evaluating the overall severity and predicting the sequelae of OSA.

\section{Integrated Grading System for OSA Severity}

As heavy reliance on a single trait in OSA to evaluate the disease severity may result in underestimation or misjudgment, a new multidimensional model to assess OSA disease severity has been elaborated. ${ }^{65}$ The frequency of respiratory events and the related acute systemic effects, such as T90, arousals, and, of equal importance, the long-term organ impact of OSA (e.g. hypertension, CVD, insulin resistance), were included in a three-dimensional model. The threedimensional volume represents a synthetic analysis of severity to better adapt to the uses for clinical management.

A refined $A B C D$ evaluation tool was also proposed based on the multi-element grading system that considers both organ damage and signs and symptoms, such as the Epworth Sleepiness Scale score, dozing episodes, self-assessed hypersomnia, and vigilance test results (Figure 1), ${ }^{65,66}$ similar to the approach in the global strategy for chronic obstructive pulmonary disease. ${ }^{67}$ The development and validation of biomarkers for these observable characteristics in OSA may be one of the priorities for future research.

\section{Deep Learning/Machine Learning}

Deep learning (DL) is a subset of machine learning and consists of various analysis methods including convolutional neural networks (CNNs) and long short-term memory (LSTM). The DL approach has been applied to
Major end organ impact: Recurrent/not well controlled

Minor end organ impact: Well controlled or No diagnosis

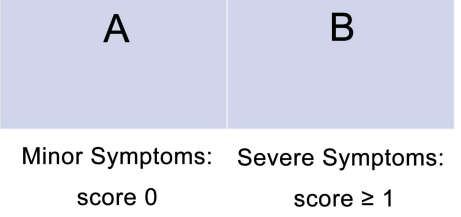

Figure I A refined $A B C D$ evaluation tool based on the multielement grading system for OSA severity.

Note: A patient is considered to presented with minor symptoms (score 0 ) if all results are negative (Epworth Sleepiness Scale $<9$, no dozing episodes or hypersomnia, negative vigilance test), or severe symptoms (score $\geq 1$ ) if any of these tests are positive. The minor end organ impact means that the following diseases, including arterial hypertension, atrial fibrillation, heart-failure, diabetes, stroke, are well controlled or never diagnosed. A patient is considered to suffer from major end organ impact if any of these diseases are recurrent or not well controlled. Thus, Patients presenting with minor symptoms (score 0 ) are classified as group $A$ or $C$, and patients suffering from minor end organ impact are classified as group A or B. Reproduced with permission from (C) ERS 2020: European Respiratory Journal 2018;52:3. doi:10.1 183/13993003.02616-2017. ${ }^{65}$

medical settings and language processing and can extract the rich data contained in PSG and automatically finish sleep staging. ${ }^{68}$ A study using three DL methods $(\mathrm{CNN}$, LSTM, CNN + LSTM) to estimate the AHI ${ }^{69}$ demonstrated the correlation $r$ value between the gold standard AHI with an estimated value of 0.84 , which showed that this system may serve as a convenient tool for a homebased sleep apnea test (HSAT). The HSAT is expected to be more efficacious and cost effective and to eventually play a crucial part in quantifying OSA in the future. Other studies have also used the DL model to predict sleep disorders in an asthma cohort ${ }^{70}$ and to predict nocturnal blood pressure in patients with OSA. ${ }^{71}$ With the rapid development of DL, it will not be long before DL methods are used widely in clinical practice.

\section{Conclusion}

It is unacceptable to acquire nothing but the AHI data from the massive amount of information stored in the PSG. Although conventional measures such as the AHI share serious limitations in various respects, novel parameters, such as the hypoxic burden and obstruction severity, are incorporating significantly more information on multidimensional traits of hypoxemia and desaturation during ventilatory disturbance. Thus, these methods are better in capturing the physiological stress and morphology in OSA and in predicting the outcomes of various conditions related to OSA. However, deficiencies 
still exist for these methods because neither the difference between the physiological impact of hypopnea versus apnea nor the arousal intensity were elucidated, and the classification of desaturation events (central or obstructive) was not considered. In addition, other new methods, such as the decreases in pulse wave amplitude derived from noninvasive photoplethysmography, are also catching the attention of researchers. ${ }^{72}$

To date, there is a scarcity of literature to clarify the widely accepted or perfect diagnostic criteria and to guide strategies on effective or precise treatment of the major neurocognitive and CVD sequelae related to OSA. To better illustrate the disease severity of OSA in the future, a more comprehensive indicator-one that considers hypoxemia, desaturation, arousal threshold, loop gain, and sleep staging, especially during the REM period-will improve the ability to determine the severity and management of OSA. With this foundation, the science in sleep and respiratory medicine will undoubtedly advance.

\section{Disclosure}

The authors report no conflicts of interest in this work.

\section{References}

1. Jordan AS, McSharry DG, Malhotra A. Adult obstructive sleep apnoea. The Lancet. 2014;383(9918):736-747. doi:10.1016/S01406736(13)60734-5

2. Malhotra A, Orr JE, Owens RL. On the cutting edge of obstructive sleep apnoea: where next? The Lancet Respir Med. 2015;3 (5):397-403

3. Levy P, Kohler M, McNicholas WT, et al. Obstructive sleep apnoea syndrome. Nat Rev Dis Primers. 2015;1:15015.

4. Benjafield AV, Ayas NT, Eastwood PR, et al. Estimation of the global prevalence and burden of obstructive sleep apnoea: a literature-based analysis. The Lancet Respir Med. 2019;7(8):687-698.

5. Punjabi NM, Newman AB, Young TB, Resnick HE, Sanders MH. Sleep-disordered breathing and cardiovascular disease: an outcome-based definition of hypopneas. Am J Respir Crit Care Med. 2008;177(10):1150-1155. doi:10.1164/rccm.200712-1884OC

6. Osman AM, Carter SG, Carberry JC, Eckert DJ. Obstructive sleep apnea: current perspectives. Nat Sci Sleep. 2018;10:21-34. doi:10.2147/NSS.S124657

7. Hillman DR, Murphy AS, Pezzullo L. The economic cost of sleep disorders. Sleep. 2006;29(3):299-305. doi:10.1093/sleep/29.3.299

8. Watson NF. Health care savings: the economic value of diagnostic and therapeutic care for obstructive sleep apnea. J Clin Sleep Med. 2016;12(8):1075-1077. doi:10.5664/jcsm.6034

9. Taranto-Montemurro L, Messineo L, Sands SA, et al. The combination of atomoxetine and oxybutynin greatly reduces obstructive sleep apnea severity. a randomized, placebo-controlled, double-blind crossover trial. Am J Respir Crit Care Med. 2019;199(10):1267-1276. doi:10.1164/rccm.201808-1493OC

10. Sands SA, Edwards BA, Terrill PI, et al. Identifying obstructive sleep apnoea patients responsive to supplemental oxygen therapy. Eur Respir J. 2018;52:3. doi:10.1183/13993003.00674-2018
11. Catalan-Serra P, Campos-Rodriguez F, Reyes-Nunez N, et al. Increased incidence of stroke, but not coronary heart disease, in elderly patients with sleep apnea. Stroke. 2019;50(2):491-494.

12. Carter SG, Carberry JC, Cho G, et al. Effect of 1 month of zopiclone on obstructive sleep apnoea severity and symptoms: a randomised controlled trial. Eur Respir J. 2018;52(1):1800149. doi:10.1183/ 13993003.00149-2018

13. Eiseman NA, Westover MB, Mietus JE, Thomas RJ, Bianchi MT. Classification algorithms for predicting sleepiness and sleep apnea severity. J Sleep Res. 2012;21(1):101-112. doi:10.1111/j.13652869.2011.00935.x

14. Young LR, Taxin ZH, Norman RG, Walsleben JA, Rapoport DM, Ayappa I. Response to CPAP withdrawal in patients with mild versus severe obstructive sleep apnea/hypopnea syndrome. Sleep. 2013;36 (3):405-412. doi:10.5665/sleep. 2460

15. Azarbarzin A, Sands SA, Stone KL, et al. The hypoxic burden of sleep apnoea predicts cardiovascular disease-related mortality: the Osteoporotic Fractures in Men Study and the Sleep Heart Health Study. Eur Heart J. 2019;40(14):1149-1157. doi:10.1093/eurheartj/ ehy624

16. Nieto FJ, Young TB, Lind BK, et al. Association of sleep-disordered breathing, sleep apnea, and hypertension in a large community-based study. Sleep Heart Health Study. JAMA. 2000;283(14):1829-1836. doi:10.1001/jama.283.14.1829

17. Punjabi NM. COUNTERPOINT: is the apnea-hypopnea index the best way to quantify the severity of sleep-disordered breathing? No. Chest. 2016;149(1):16-19. doi:10.1378/chest.14-2261

18. Kendzerska T, Leung RS. Going beyond the apnea-hypopnea index. Chest. 2016;149(5):1349-1350. doi:10.1016/j.chest.2016.02.671

19. Sánchez-de-la-Torre M, Sánchez-de-la-Torre A, Bertran S, et al. Effect of obstructive sleep apnoea and its treatment with continuous positive airway pressure on the prevalence of cardiovascular events in patients with acute coronary syndrome (ISAACC study): a randomised controlled trial. The Lancet Respir Med. 2020;8 (4):359-367. doi:10.1016/S2213-2600(19)30271-1

20. McEvoy RD, Antic NA, Heeley E, et al. CPAP for prevention of cardiovascular events in obstructive sleep apnea. $N$ Engl $J$ Med. 2016;375(10):919-931. doi:10.1056/NEJMoa1606599

21. Sharma S, Fox H, Aguilar F, et al. Auto positive airway pressure therapy reduces pulmonary pressures in adults admitted for acute heart failure with pulmonary hypertension and obstructive sleep apnea. The ASAP-HF Pilot Trial Sleep. 2019;42:7.

22. Kohler M, Stradling JR. Mechanisms of vascular damage in obstructive sleep apnea. Nat Rev Cardiol. 2010;7(12):677-685. doi:10.1038/ nrcardio.2010.145

23. Parati G, Lombardi C, Hedner J, et al. Position paper on the management of patients with obstructive sleep apnea and hypertension: joint recommendations by the European Society of Hypertension, by the European Respiratory Society and by the members of European COST (COoperation in Scientific and Technological research) ACTION B26 on obstructive sleep apnea. J Hypertens. 2012;30 (4):633-646.

24. Marin JM, Carrizo SJ, Vicente E, Agusti AGN. Long-term cardiovascular outcomes in men with obstructive sleep apnoea-hypopnoea with or without treatment with continuous positive airway pressure: an observational study. The Lancet. 2005;365(9464):1046-1053. doi:10.1016/S0140-6736(05)71141-7

25. Patil SP, Ayappa IA, Caples SM, Kimoff RJ, Patel SR, Harrod CG. Treatment of adult obstructive sleep apnea with positive airway pressure: an american academy of sleep medicine clinical practice guideline. J Clin Sleep Med. 2019;15(2):335-343. doi:10.5664/ jesm. 7640

26. Zhao YY, Javaheri S, Wang R, et al. Associations between sleep apnea and subclinical carotid atherosclerosis: the multi-ethnic study of atherosclerosis. Stroke. 2019;50(12):3340-3346. doi:10.1161/ STROKEAHA.118.022184 
27. Oldenburg O, Wellmann B, Buchholz A, et al. Nocturnal hypoxaemia is associated with increased mortality in stable heart failure patients. Eur Heart J. 2016;37(21):1695-1703. doi:10.1093/eurheartj/ehv624

28. Oldenburg O, Costanzo MR, Germany R, McKane S, Meyer TE, Fox H. Improving nocturnal hypoxemic burden with transvenous phrenic nerve stimulation for the treatment of central sleep apnea. J Cardiovasc Transl Res. 2020. doi:10.1007/s12265-020-10061-0

29. Asha'ari ZA, Rahman JA, Mohamed AH, Abdullah K, Leman WI. Association between severity of obstructive sleep apnea and number and sites of upper airway operations with surgery complications. JAMA Otolaryngol Head Neck Surg. 2017;143(3):239-246. doi:10.1001/jamaoto.2016.3268

30. Ravesloot MJ, van Maanen JP, Hilgevoord AA, van Wagensveld BA, de Vries N. Obstructive sleep apnea is underrecognized and underdiagnosed in patients undergoing bariatric surgery. Eur Arch Otorhinolaryngol. 2012;269(7):1865-1871. doi:10.1007/s00405-012-1948-0

31. Unosawa S, Sezai A, Akahoshi T, et al. Arrhythmia and sleep-disordered breathing in patients undergoing cardiac surgery. J Cardiol. 2012;60(1):61-65. doi:10.1016/j.jjcc.2012.01.012

32. Siwasaranond N, Nimitphong H, Manodpitipong A, et al. The relationship between diabetes-related complications and obstructive sleep apnea in Type 2 diabetes. J Diabetes Res. 2018;2018:9269170. doi:10.1155/2018/9269170

33. Hui P, Zhao L, Xie Y, et al. Nocturnal hypoxemia causes hyperglycemia in patients with obstructive sleep apnea and Type 2 diabetes mellitus. Am J Med Sci. 2016;351(2):160-168. doi:10.1016/j. amjms.2015.12.002

34. Myllymaa K, Myllymaa S, Leppanen T, et al. Effect of oxygen desaturation threshold on determination of OSA severity during weight loss. Sleep Breath. 2016;20(1):33-42. doi:10.1007/s11325015-1180-4

35. Kirby SC, Anderson WM, Chesson AL, George RB. Computer quantitation of saturation impairment time as an index of oxygenation during sleep. Comput Methods Programs Biomed. 1992;38(2-3):107-115. doi:10.1016/0169-2607(92)90081-H

36. Chesson AL, McDowell Anderson W, Walls RC, Bairnsfather LE. Comparison of two methods of quantitative assessment of hypoxemia in patients with sleep disorders. Sleep Med. 2001;2(1):37-45. doi:10.1016/S1389-9457(00)00041-1

37. Kim JS, Azarbarzin A, Wang R, et al. Association of novel measures of sleep disturbances with blood pressure: the multi-ethnic study of atherosclerosis. Thorax. 2020;75(1):57-63. doi:10.1136/thoraxjnl2019-213533

38. Azarbarzin A, Sands SA, Taranto-Montemurro L, et al. The sleep apnea-specific hypoxic burden predicts incident heart failure. Chest. 2020;158(2):739-750. doi:10.1016/j.chest.2020.03.053

39. Linz D, Baumert M. How to assess nocturnal hypoxaemic burden in Cardiology? Eur Heart J. 2019;40(35):2988. doi:10.1093/eurheartj/ ehz272

40. Butler MP, Emch JT, Rueschman M, et al. Apnea-hypopnea event duration predicts mortality in men and women in the sleep heart health study. Am J Respir Crit Care Med. 2019;199(7):903-912. doi:10.1164/rccm.201804-07580C

41. Kulkas A, Tiihonen P, Julkunen P, Mervaala E, Toyras J. Novel parameters indicate significant differences in severity of obstructive sleep apnea with patients having similar apnea-hypopnea index. Med Biol Eng Comput. 2013;51(6):697-708. doi:10.1007/s11517-0131039-4

42. Leppanen T, Toyras J, Mervaala E, Penzel T, Kulkas A. Severity of individual obstruction events increases with age in patients with obstructive sleep apnea. Sleep Med. 2017;37:32-37. doi:10.1016/j. sleep.2017.06.004

43. Muraja-Murro A, Kulkas A, Hiltunen M, et al. The severity of individual obstruction events is related to increased mortality rate in severe obstructive sleep apnea. J Sleep Res. 2013;22(6):663-669. doi:10.1111/jsr.12070
44. Kulkas A, Leppanen T, Sahlman J, et al. Novel parameters reflect changes in morphology of respiratory events during weight loss. Physiol Meas. 2013;34(9):1013-1026. doi:10.1088/0967-3334/34/9/1013

45. Muraja-Murro A, Kulkas A, Hiltunen M, et al. Adjustment of apnea-hypopnea index with severity of obstruction events enhances detection of sleep apnea patients with the highest risk of severe health consequences. Sleep Breath. 2014;18(3):641-647. doi:10.1007/ s11325-013-0927-z

46. Azarbarzin A, Sands SA, White DP, Redline S, Wellman A. The hypoxic burden: a novel sleep apnoea severity metric and a predictor of cardiovascular mortality-Reply to 'The hypoxic burden: also known as the desaturation severity parameter'. Eur Heart J. 2019;40(35):2994-2995. doi:10.1093/eurheartj/ehz273

47. Linz D, Colling S, Nussstein W, et al. Nocturnal hypoxemic burden is associated with epicardial fat volume in patients with acute myocardial infarction. Sleep Breath. 2018;22(3):703-711. doi:10.1007/ s11325-017-1616-0

48. Khoshkish S, Hohl M, Linz B, et al. The association between different features of sleep-disordered breathing and blood pressure: a cross-sectional study. J Clin Hypertens (Greenwich). 2018;20 (3):575-581. doi:10.1111/jch.13202

49. Pincus SM. Assessing serial irregularity and its implications for health. Ann N Y Acad Sci. 2001;954:245-267. doi:10.1111/j.17496632.2001.tb02755.x

50. Nakayama H, Hokari S, Ohshima Y, Matsuto T, Shimohata T. Breathing irregularity is independently associated with the severity of obstructive sleep apnea in patients with multiple system atrophy. J Clin Sleep Med. 2018;14(10):1661-1667. doi:10.5664/ jcsm. 7368

51. Liu J, Huang R, Xiao Y, Lin S. ApEn for assessing hypoxemia severity in obstructive sleep apnea hypopnea syndrome patients. Sleep Breath. 2020.

52. Del Campo F, Hornero R, Zamarron C, Abasolo DE, Alvarez D. Oxygen saturation regularity analysis in the diagnosis of obstructive sleep apnea. Artif Intell Med. 2006;37(2):111-118. doi:10.1016/j. artmed.2005.10.005

53. Younes M. CrossTalk proposal: elevated loop gain is a consequence of obstructive sleep apnoea. J Physiol. 2014;592(14):2899-2901. doi:10.1113/jphysiol.2014.271833

54. Loewen A, Ostrowski M, Laprairie J, et al. Determinants of ventilatory instability in obstructive sleep apnea: inherent or acquired? Sleep. 2009;32(10):1355-1365. doi:10.1093/sleep/32.10.1355

55. de Chazal P, Sutherland K, Cistulli PA. Advanced polysomnographic analysis for OSA: a pathway to personalized management? Respirology. 2020;25(3):251-258. doi:10.1111/resp.13564

56. Thomas RJ, Mietus JE, Peng CK, Goldberger AL. An electrocardiogram-based technique to assess cardiopulmonary coupling during sleep. Sleep. 2005;28(9):1151-1161. doi:10.1093/sleep/ 28.9.1151

57. Cho JH, Kim HJ. The effect of continuous positive airway pressure on cardiopulmonary coupling. Sleep and Breathing. 2017;21 (2):341-345. doi:10.1007/s11325-016-1419-8

58. Utriainen KT, Airaksinen JK, Polo OJ, et al. Alterations in heart rate variability in patients with peripheral arterial disease requiring surgical revascularization have limited association with postoperative major adverse cardiovascular and cerebrovascular events. PLoS One. 2018;13(9):e0203519. doi:10.1371/journal. pone. 0203519

59. Hillebrand S, Gast KB, de Mutsert R, et al. Heart rate variability and first cardiovascular event in populations without known cardiovascular disease: meta-analysis and dose-response meta-regression. Europace. 2013;15(5):742-749. doi:10.1093/europace/eus341

60. Makikallio TH, Barthel P, Schneider R, et al. Prediction of sudden cardiac death after acute myocardial infarction: role of Holter monitoring in the modern treatment era. Eur Heart J. 2005;26 (8):762-769. doi:10.1093/eurheartj/ehi188 
61. Nastalek P, Bochenek G, Kania A, Celejewska-Wojcik N, Mejza F, Sladek K. Heart Rate Variability in the Diagnostics and CPAP Treatment of Obstructive Sleep Apnea. Adv Exp Med Biol. 2019;1176:25-33.

62. Younes M, Ostrowski M, Soiferman M, et al. Odds ratio product of sleep EEG as a continuous measure of sleep state. Sleep. 2015;38 (4):641-654. doi:10.5665/sleep.4588

63. Mann DL, Terrill PI, Azarbarzin A, et al. Quantifying the magnitude of pharyngeal obstruction during sleep using airflow shape. Eur Respir J. 2019;54:1. doi:10.1183/13993003.02262-2018

64. Wiriyaporn D, Wang L, Aboussouan LS. Expiratory time constant and sleep apnea severity in the overlap syndrome. J Clin Sleep Med. 2016;12(3):327-332. doi:10.5664/jcsm.5576

65. Randerath W, Bassetti CL, Bonsignore MR, et al. Challenges and perspectives in obstructive sleep apnoea: report by an ad hoc working group of the Sleep Disordered Breathing Group of the European Respiratory Society and the European Sleep Research Society. Eur Respir J. 2018;52:3. doi:10.1183/13993003.02616-2017

66. Crinion SJ, Ryan S, McNicholas WT. Obstructive sleep apnoea as a cause of nocturnal nondipping blood pressure: recent evidence regarding clinical importance and underlying mechanisms. Eur Respir J. 2017;49:1. doi:10.1183/13993003.01818-2016

67. Singh D, Agusti A, Anzueto A, et al. Global strategy for the diagnosis, management, and prevention of chronic obstructive lung disease: the GOLD science committee report 2019. Eur Respir J. 2019;53:5. doi:10.1183/13993003.00164-2019
68. Hong S, Zhou Y, Shang J, Xiao C, Sun J. Opportunities and challenges of deep learning methods for electrocardiogram data: A systematic review. Comput Biol Med. 2020;122:103801. doi:10. 1016/j.compbiomed.2020.103801

69. Hafezi M, Montazeri N, Zhu K, Alshaer H, Yadollahi A, Taati B. Sleep apnea severity estimation from respiratory related movements using deep Learning. Conf Proc IEEE Eng Med Biol Soc. 2019;2019:1601-1604.

70. Phan DV, Yang NP, Kuo CY, Chan CL. Deep learning approaches for sleep disorder prediction in an asthma cohort. J Asthma. 2020;1-9. doi:10.1080/02770903.2020.1742352

71. Prasad B, Agarwal C, Schonfeld E, Schonfeld D, Mokhlesi B. Deep learning applied to polysomnography to predict blood pressure in obstructive sleep apnea and obesity hypoventilation: a proof-ofconcept study. J Clin Sleep Med. 2020;16(10):1797-1803. doi:10. $5664 /$ jcsm. 8608

72. Hirotsu C, Betta M, Bernardi G, et al. Pulse wave amplitude drops during sleep: clinical significance and characteristics in a general population sample. Sleep. 2020;43:7. doi:10.1093/sleep/zsz322

\section{Publish your work in this journal}

Nature and Science of Sleep is an international, peer-reviewed, open access journal covering all aspects of sleep science and sleep medicine, including the neurophysiology and functions of sleep, the genetics of sleep, sleep and society, biological rhythms, dreaming, sleep disorders and therapy, and strategies to optimize healthy sleep.
The manuscript management system is completely online and includes a very quick and fair peer-review system, which is all easy to use. Visit http://www.dovepress.com/testimonials.php to read real quotes from published authors. 